\section{Fatores associados ao aleitamento materno exclusivo: o papel do cuidado na atenção básica}

\author{
Factors associated with exclusive breastfeeding: \\ the role of primary health care
}

\author{
1 Universidade Federal \\ Fluminense, Niterói, Brasil. \\ 2 Escola Nacional de Saúde \\ Pública Sergio Arouca, \\ Fundação Oswaldo Cruz, Rio \\ de Janeiro, Brasil. \\ Correspondência \\ R. S. V. Pereira \\ Universidade Federal \\ Fluminense. \\ Rua Marquês de Paraná 303 \\ prédio anexo, 30 andar, Niterói \\ RJ 24030-210, Brasil. \\ rosanesvp@uol.com.br
}

\begin{abstract}
The aim of this study was to assess the association between promotion, protection, and support for breastfeeding in primary health care services and prevalence of exclusive breastfeeding in the first six months of life. A cross-sectional study was conducted in a representative sample of 1,029 mothers of infants younger than six months treated at primary health care facilities in the city of Rio de Janeiro, in southeastern Brazil. Prevalence ratios (PR) were estimated by Poisson regression. Prevalence of exclusive breastfeeding was 58.1\%. Factors associated with increased prevalence of exclusive breastfeeding were: white skin color $(P R=$ 1.20; 95\%CI: 1.05-1.36); schooling ( $P R=1.19$; 95\%CI: 1.05-1.35); marital status (married or in common-law marriage) ( $P R=1.72 ; 95 \%$ IC:1.022.90); previous breastfeeding $(P R=1.27$; 95\%CI: 1.08-1,49); exclusive breastfeeding at the time of hospital discharge ( $P R=2.01$; 95\%CI: 1.20-3.36); group support for the mother $(P R=1.14 ; 95 \% C I$ : 1.01-1.28); and orientation on breastfeeding $(P R=$ 1.20; 95\%CI: 1.08-1.33). Prevalence of exclusive breastfeeding decreased $17 \%$ per month in the infant's life. Support groups and orientation by primary health care for breastfeeding were associated with increased exclusive breastfeeding prevalence.
\end{abstract}

Breast Feeding; Primary Health Care; Cross-Sectional Studies
Rosane Siqueira Vasconcellos Pereira 1 Maria Inês Couto de Oliveira 1 Carla Lourenço Tavares de Andrade 2 Alexandre dos Santos Brito ${ }^{1}$

\section{Introdução}

A amamentação tem desempenhado um papel importante na saúde da mulher e da criança e inúmeros trabalhos têm sido publicados ressaltando a proteção conferida à criança, a curto e longo prazo 1,2. O leite humano é considerado o padrão ouro na alimentação do lactente e o crescimento e desenvolvimento da criança amamentada, a norma 2,3 .

Em 2001, em razão das evidências da superioridade do leite humano, a Organização Mundial da Saúde (OMS) passou a adotar como recomendação o aleitamento materno exclusivo por seis meses 4 . A OMS definiu também como indicadores: aleitamento materno exclusivo (criança recebeu apenas leite materno, diretamente do peito ou ordenhado, podendo também estar recebendo medicamentos, vitaminas ou minerais), aleitamento materno predominante (criança recebeu predominantemente o leite materno e, também, outros líquidos, como água, chá ou suco), aleitamento materno complementado (criança recebeu leite materno e outros alimentos) e aleitamento materno (criança recebeu leite materno - engloba todas as possibilidades anteriores) 5 .

As ações de promoção, proteção e apoio ao aleitamento materno têm se mostrado importantes para a melhoria da saúde da criança e também ações estratégicas para a organização e qualificação dos serviços. Ações desenvolvidas 
nos hospitais vêm sendo reconhecidas como de fundamental importância para o início da amamentação ${ }^{6}$. Trabalhos demonstram a efetividade da Iniciativa Hospital Amigo da Criança (IHAC) em mudar as rotinas hospitalares pela promoção de práticas facilitadoras da amamentação 7 .

Uma revisão sistemática identificou ações efetivas na promoção, proteção e apoio à amamentação na rede básica de saúde ${ }^{8}$ e se tornou a base para a criação da Iniciativa Unidade Básica Amiga da Amamentação (IUBAAM) que propõe o cumprimento de Dez Passos para o Sucesso da Amamentação ${ }^{9}$. Dentre esses passos, destacamse a capacitação dos profissionais, as orientações sobre o manejo da amamentação e os grupos de apoio à amamentação com gestantes e mães. Ações integradas, compreendendo o pré-natal, assistência ao parto e pós-parto, com apoio contínuo, apresentam um efeito sinérgico melhorando a qualidade da assistência à mulher que amamenta 10 .

Em 2008, o Ministério da Saúde lançou uma nova estratégia de promoção ao aleitamento materno na atenção básica, por meio da revisão do processo de trabalho interdisciplinar nas unidades básicas de saúde, apoiada nos princípios da educação permanente em saúde: a Rede Amamenta Brasil, que está em fase de implantação nos estados e municípios 11 .

Considerando o papel do aleitamento materno na redução da morbimortalidade infantil 12 , as iniciativas de promoção, proteção e apoio ao aleitamento materno realizadas nos hospitais e na atenção básica vêm sendo consideradas prioritárias pela Política Nacional de Saúde da Criança/Aleitamento Materno 11. O monitoramento da prevalência de aleitamento materno representa uma das estratégias de avaliação do cuidado à infância em nosso país 11 .

Pesquisas apontam que a prevalência de aleitamento materno exclusivo no Brasil está ainda muito aquém das recomendações. Relatório do Ministério da Saúde, com dados da Pesquisa $\mathrm{Na}$ cional de Demografia e Saúde da Criança e da Mulher (PNDS-2006), realizada em 2006, mostra uma prevalência de aleitamento materno exclusivo entre menores de seis meses de 38,6\% 13 . De acordo com a II Pesquisa de Prevalência de Aleitamento Materno nas Capitais Brasileiras e Distrito Federal 14, realizada em 2008, a prevalência de aleitamento materno exclusivo nos primeiros seis meses de vida é de $41 \%$.

No Município do Rio de Janeiro, a prevalência de aleitamento materno exclusivo está em ascensão, paralelamente ao implemento das ações de promoção, proteção e apoio à amamentação nos hospitais e rede básica de saúde 15 . A prevalência de aleitamento materno exclusivo em meno- res de seis meses variou de 13,8\% em 1996 para $33,3 \%$ em 2006, com aumento significativo no período $(\mathrm{p}=0,000)$, mas ainda abaixo dos índices nacionais de 2006 (38,6\%) 13 .

No Município do Rio de Janeiro, várias unidades de saúde vêm sendo credenciadas como Hospital Amigo da Criança 7e Unidade Básica Amiga da Amamentação 9, participando juntamente com os Bancos de Leite Humano 16, de uma rede integrada de cuidados que busca melhorar os indicadores da atenção materno-infantil e aumentar a prevalência de aleitamento materno. As unidades básicas de saúde apresentam diferentes graus de desenvolvimento dessas ações, com impacto sobre o processo de trabalho e produção do cuidado. A avaliação das ações subsidia o planejamento de estratégias de qualificação da atenção básica 17 .

Vários trabalhos têm sido publicados sobre as ações que vêm sendo desenvolvidas para promover, proteger e apoiar a amamentação, entretanto ainda são poucos os estudos que avaliaram a associação entre as intervenções desenvolvidas na atenção básica e a prevalência de aleitamento materno exclusivo nos primeiros seis meses de vida $8,17,18,19$

A prevalência ainda baixa de aleitamento materno exclusivo em nosso país aponta que novas abordagens devem ser pensadas, valorizando as ações efetivas na promoção, proteção e apoio ao aleitamento materno, mas também o contexto de processo de trabalho em que elas acontecem.

Este estudo busca analisar os fatores associados ao aleitamento materno exclusivo, particularmente as ações de promoção, proteção e apoio à amamentação desenvolvidas com mães e crianças menores de seis meses assistidas nas unidades básicas de saúde do Município do Rio de Janeiro.

\section{Metodologia}

Para o desenvolvimento deste estudo, foi utilizada a base de dados do projeto interinstitucional: Avaliação da Qualidade da Assistência à Criança Menor de Seis Meses Prestada por Unidades Básicas do Sistema Único de Saúde do Município do Rio de Janeiro, coordenado pela Escola Nacional de Saúde Pública Sergio Arouca, Fundação Oswaldo Cruz (ENSP/Fiocruz), que contou com financiamento do Programa Estratégico de Apoio à Pesquisa em Saúde (PAPES), da Fundação de Amparo à Pesquisa do Estado do Rio de Janeiro (FAPERJ) e do Conselho Nacional de Desenvolvimento Científico e Tecnológico (CNPq).

Este projeto, de desenho transversal, foi realizado em uma amostra representativa das unida- 
des básicas do Sistema Único de Saúde (SUS) do Município do Rio de Janeiro e das crianças menores de seis meses acompanhadas nessas unidades. A amostra foi realizada em dois estágios: o primeiro constituído das unidades básicas de saúde (UBS) e o segundo das crianças.

As unidades de saúde foram selecionadas de forma sistemática, em caracol, pela combinação de dois fatores, a saber: a distância euclidiana, calculada pelas coordenadas geográficas dos estabelecimentos de saúde em relação ao Centro Administrativo do Município do Rio de Janeiro e a freqüência acumulada do número médio mensal de consultas em crianças menores de seis meses de idade no primeiro semestre de 2005 20. Assumiu-se que quanto mais próxima ao Centro Administrativo do Município do Rio de Janeiro fosse a unidade de saúde, melhor seria a qualidade da atenção à criança menor de seis meses de idade. As unidades foram ordenadas segundo as distâncias do Centro, sendo produzida uma amostra geograficamente representativa do Município do Rio de Janeiro. A probabilidade de cada unidade participar da amostra foi proporcional ao volume de consultas pediátricas realizadas.

O tamanho amostral foi calculado assumindo-se uma prevalência de inadequação do cuidado à criança de 50\%; uma precisão relativa de $13 \%$ do estimador e um nível de $95 \%$ de confiança. O tamanho da amostra foi calculado em 1.080 mães de crianças menores de seis meses, e, em cada uma das 27 unidades básicas de saúde amostradas, foram realizadas 40 entrevistas. As mães de bebês menores de seis meses a serem consultados em cada turno de atendimento eram listadas. O entrevistador, então, procedia à seleção das mães mediante consulta a uma tabela com números aleatórios cuja ordem variava segundo o número de bebês a serem consultados, o turno e o dia da semana.

Foi realizado um estudo piloto em três unidades básicas de saúde de diferentes tipos para teste dos instrumentos e definição da logística do trabalho de campo. Foi utilizado um questionário estruturado que versava sobre características maternas e do bebê e sobre as várias ações da atenção integral à saúde da criança. Para a coleta dos dados referentes ao tipo de aleitamento materno praticado pela criança, foi utilizado o recordatório das últimas 24 horas - current status 21 . Os dados foram obtidos por meio de entrevistas diárias, nos períodos da manhã e da tarde, realizadas após a consulta médica ou de enfermeiro, com mães de crianças menores de seis meses. A coleta de dados foi realizada entre os meses de julho e novembro de 2007 por profissionais e estudantes da área de saúde previamente treinados e sob supervisão.
O estudo foi aprovado pelos Comitês de Ética da ENSP/Fiocruz (parecer n ${ }^{\circ}$. 132/06 de 7 de março de 2007) e da Secretaria Municipal de Saúde do Rio de Janeiro (parecer no. 74A/2007 de 18 de junho de 2007). As entrevistas foram realizadas mediante assinatura de termo de consentimento livre e esclarecido.

Foram incluídas UBS como Policlínicas, Postos de Assistência Médica (PAM), Centros Municipais de Saúde, Postos de Saúde, Unidades de Atenção de Cuidados Primários à Saúde e Programa de Agentes Comunitários de Saúde (PACS). Foram excluídos os Postos de Saúde da Família por não se dispor de um sistema de informação atualizado quanto ao número de crianças menores de seis meses atendidos por eles e pela baixa representatividade da rede de postos de saúde da família na atenção básica do Município do Rio de Janeiro (http://www.saude.rio.rj.gov.br, acessado em 26/Nov/2008). Para o presente estudo, foram excluídas as crianças filhas de mães HIV positivas, pois para elas o aleitamento materno é contra-indicado 22, e aquelas que chegaram à unidade básica de saúde já no primeiro atendimento sem estarem sendo amamentadas, restando 1.029 crianças para análise.

A Figura 1 elucida as variáveis pesquisadas, dentro de um modelo teórico de determinação do desfecho deste estudo, o aleitamento materno exclusivo: (1) características maternas - idade, cor, escolaridade, trabalho remunerado, número de bens, situação conjugal, paridade, experiência pregressa com amamentação; (2) características do bebê e da assistência pré-natal e ao parto sexo do bebê, baixo peso ao nascer, idade do bebê, realização de pré-natal, certificação do local de ocorrência do parto como Hospital Amigo da Criança, tipo de parto, aleitamento materno exclusivo na alta hospitalar; (3) características da unidade básica de saúde e das orientações de aleitamento materno - tipo de unidade, ter recebido orientação de amamentação no primeiro dia em que veio na UBS, ter sido falado sobre amamentação na consulta, ter sido falado sobre amamentação em grupo, ter sido falado sobre amamentação de outro jeito (na visita domiciliar, no acolhimento, na sala de vacina ou outro), ter sido mostrado como colocar o bebê para mamar, ter sido explicado sobre livre demanda, sobre como realizar ordenha manual, que não se deve dar mamadeira, até quando o aleitamento materno deve ser exclusivo, número de orientações recebidas na UBS, satisfação das mães com o apoio recebido para amamentar e qualidade do acompanhamento do bebê.

Inicialmente, foi desenvolvida uma análise univariada para conhecimento do perfil das mães e das crianças. Em seguida foi realizada 
Modelo teórico de determinação do aleitamento materno exclusivo em crianças assistidas em unidades básicas de saúde (UBS) no Município do Rio de Janeiro, Brasil, 2007.

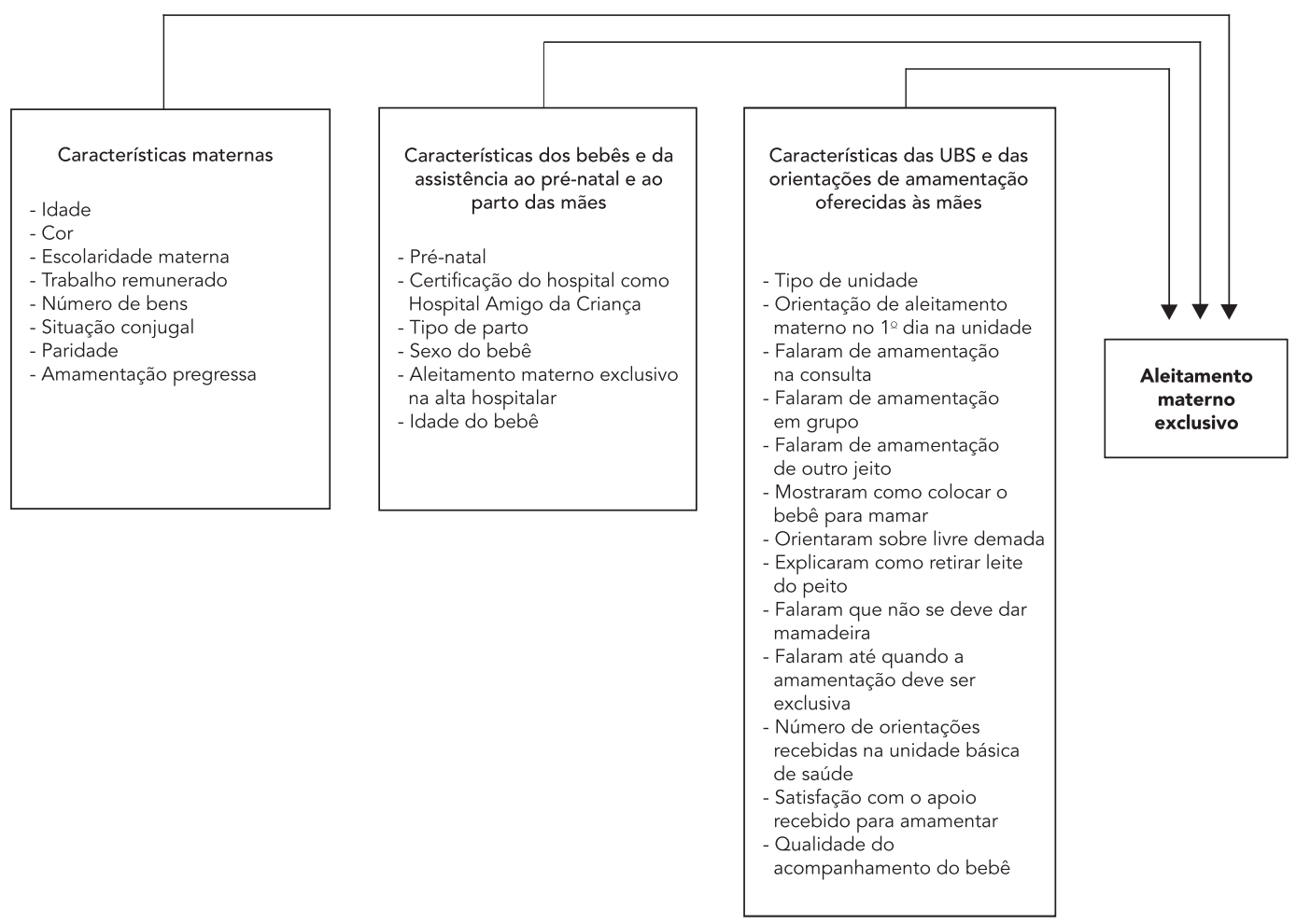

uma análise bivariada entre cada variável de exposição, expressa de forma dicotômica, e o aleitamento materno exclusivo, sendo descritas as distribuições de aleitamento materno exclusivo segundo as categorias das variáveis de exposição. Foram também realizados testes de hipóteses de qui-quadrado e obtidas razões de prevalência (RP) brutas com seus respectivos intervalos de 95\% de confiança (IC95\%).

Variáveis de exposição que, na análise bivariada, mostraram-se associadas com o aleitamento materno exclusivo com nível de significância observado menor ou igual a $20 \%$ no teste de quiquadrado (valor de $\mathrm{p} \leq 0,20$ ) foram selecionadas para a análise multivariada realizada por modelo de regressão de Poisson com variância robusta. Tal modelo foi utilizado por se tratar de estudo transversal com desfecho não raro 23 .

Todo o processo de modelagem foi ajustado pela idade da criança, como variável contínua. A análise multivariada parte do modelo de regressão de Poisson saturado, que contém todas as variáveis de exposição selecionadas na análise bivariada. Durante o processo de modelagem, foram eliminadas do modelo (backward elimination) aquelas variáveis que apresentavam nível de significância observado superior a 5\% no teste de Wald. Desse modo, o modelo final, utilizado para estimar medidas de associação de razão de prevalência ajustada, com seus respectivos IC95\%, foi composto por variáveis de exposição que mantiveram nível de $5 \%$ de significância.

Como foi utilizado um desenho complexo de amostragem para seleção de amostra representativa da população, foi necessário que a análise estatística dos dados incorporasse o uso da ponderação e o desenho amostral 24, o que foi realizado por meio do procedimento survey, que considera os dois estágios de seleção da amostra. O programa Stata 9.0 (Stata Corp., College Station, Estados Unidos) foi utilizado para a análise. 


\section{Resultados}

Das 1.082 mães entrevistadas, foram excluídas quatro por serem HIV positivas e 49 por não estarem amamentando ao chegarem pela primeira vez na unidade, restando 1.029 mães para análise. No momento da entrevista, $58,1 \%$ dos bebês estavam em aleitamento materno exclusivo, $10,7 \%$ em aleitamento materno predominante, $24,1 \%$ em aleitamento materno complementado. Não estavam mais sendo amamentados 7,1\% dos bebês (dados não apresentados em tabela).

Quanto às características das entrevistadas, $78,5 \%$ das mães eram adultas, $27,8 \%$ declararam- se de cor branca, apenas $25,6 \%$ apresentaram pelo menos o ensino médio completo, a maioria não exercia trabalho remunerado (65,5\%) e 44,3\% dispunha de 5 a 10 bens no domicílio. Tinham companheiro $85,9 \%$ das mães, $55,2 \%$ tinham dois ou mais filhos e $31,8 \%$ haviam amamentado anteriormente por mais de seis meses. Na análise bivariada, todas essas variáveis mostraram-se associadas ao desfecho com nível de significância observado menor ou igual a $20 \%$ (Tabela 1 ).

Na Tabela 2, pode-se observar que $49,4 \%$ dos bebês eram do sexo feminino e 8,3\% apresentaram baixo peso ao nascer. Fizeram pré-natal $98,6 \%$ das mães, a maioria $(67,0 \%)$ das mães teve

Prevalência e razão de prevalência (RP) bruta de aleitamento materno exclusivo segundo características das mães de crianças menores de seis meses assistidas nas unidades básicas de saúde (UBS) do Município do Rio de Janeiro, Brasil, 2007.

\begin{tabular}{|c|c|c|c|c|c|c|}
\hline \multirow[t]{2}{*}{ Variáveis } & \multicolumn{2}{|c|}{ Freqüência } & \multicolumn{4}{|c|}{ Aleitamento materno exclusivo } \\
\hline & $\mathbf{n}$ & $\%$ & $\%$ & $\begin{array}{c}\text { RP } \\
\text { bruta }\end{array}$ & IC95\% & $\begin{array}{l}\text { Valor } \\
\text { de } p\end{array}$ \\
\hline \multicolumn{7}{|l|}{ Idade das mães (anos) [ $N=1.029]$} \\
\hline $20-44$ & 808 & 78,5 & 59,5 & 1,13 & $0,96-1,33$ & 0,108 \\
\hline $12-19$ & 221 & 21,5 & 52,5 & 1,00 & & \\
\hline \multicolumn{7}{|l|}{ Cor $[N=1.029]$} \\
\hline Branca & 286 & 27,8 & 62,2 & 1,11 & $0,99-1,24$ & 0,086 \\
\hline Não branca & 743 & 72,2 & 56,3 & 1,00 & & \\
\hline \multicolumn{7}{|l|}{ Escolaridade [N = 1.029] } \\
\hline Ensino Médio completo e mais & 263 & 25,6 & 62,7 & 1,11 & $0,97-1,27$ & 0,120 \\
\hline Até Ensino Médio incompleto & 766 & 74,4 & 56,4 & 1,00 & & \\
\hline \multicolumn{7}{|l|}{ Trabalho remunerado [ $\mathrm{N}=1.029]$} \\
\hline Não & 674 & 65,5 & 60,3 & 1,13 & $1,01-1,26$ & 0,030 \\
\hline Sim & 355 & 34,5 & 53,5 & 1,00 & & \\
\hline \multicolumn{7}{|l|}{ Número de bens [ $\mathrm{N}=1.029]$} \\
\hline $5-10$ & 456 & 44,3 & 56,1 & 0,95 & $0,88-1,02$ & 0,121 \\
\hline $1-4$ & 573 & 55,7 & 59,4 & 1,00 & & \\
\hline \multicolumn{7}{|l|}{ Situação conjugal [N = 1.029] } \\
\hline Tem companheiro & 884 & 85,9 & 60,8 & 1,47 & $1,14-1,90$ & 0,001 \\
\hline Não tem companheiro & 145 & 14,1 & 41,3 & 1,00 & & \\
\hline \multicolumn{7}{|l|}{ Paridade [N = 1.026] } \\
\hline 2 ou mais filhos & 566 & 55,2 & 60,0 & 1,10 & $0,95-1,27$ & 0,190 \\
\hline Primípara & 460 & 44,8 & 54,6 & 1,00 & & \\
\hline \multicolumn{7}{|l|}{ Amamentação pregressa [ $\mathrm{N}=1.022]$} \\
\hline 7-84 meses & 325 & 31,8 & 64,7 & 1,19 & $1,04-1,38$ & 0,020 \\
\hline $0-6$ e 29 dias & 697 & 68,2 & 54,2 & 1,00 & & \\
\hline
\end{tabular}

IC95\%: intervalo de 95\% de confiança. 
Tabela 2

Prevalência e razão de prevalência (RP) bruta de aleitamento materno exclusivo segundo características dos bebês e da assistência ao pré-natal e ao parto das mães de crianças menores de seis meses assistidas nas unidades básicas de saúde (UBS) do Município do Rio de Janeiro, Brasil, 2007.

\begin{tabular}{|c|c|c|c|c|c|c|}
\hline \multirow[t]{2}{*}{ Variáveis } & \multicolumn{2}{|c|}{ Freqüência } & \multicolumn{4}{|c|}{ Aleitamento materno exclusivo } \\
\hline & $\mathbf{n}$ & $\%$ & $\%$ & $\begin{array}{c}\text { RP } \\
\text { bruta }\end{array}$ & IC95\% & $\begin{array}{l}\text { Valor } \\
\text { de } p\end{array}$ \\
\hline \multicolumn{7}{|l|}{ Sexo do bebê [n = 1.029] } \\
\hline Feminino & 508 & 49,4 & 61,0 & 1,11 & $0,97-1,28$ & 0,124 \\
\hline Masculino & 521 & 50,6 & 54,7 & 1,00 & & \\
\hline \multicolumn{7}{|l|}{ Baixo peso ao nascer $[n=1.020]$} \\
\hline Não & 935 & 91,7 & 58,2 & 1,12 & $0,90-1,40$ & 0,262 \\
\hline Sim & 85 & 8,3 & 51,9 & 1,00 & & \\
\hline \multicolumn{7}{|l|}{ Fez pré-natal [ $n=1.029]$} \\
\hline Não & 14 & 1,4 & 49,7 & 0,86 & $0,56-1,32$ & 0,431 \\
\hline Sim & 1015 & 98,6 & 58,0 & 1,00 & & \\
\hline \multicolumn{7}{|l|}{ Certificação do hospital [n = 1.029] } \\
\hline Não Hospital Amigo da Criança & 340 & 33,0 & 55,4 & 0,93 & $0,79-1,10$ & 0,384 \\
\hline Hospital Amigo da Criança ou em processo & 689 & 67,0 & 59,5 & 1,00 & & \\
\hline \multicolumn{7}{|l|}{ Tipo de parto [ $\mathrm{n}=1.029]$} \\
\hline Normal & 655 & 63,7 & 60,7 & 1,15 & $0,96-1,37$ & 0,118 \\
\hline Cesárea & 374 & 36,3 & 53,0 & 1,00 & & \\
\hline \multicolumn{7}{|c|}{$\begin{array}{l}\text { Aleitamento materno exclusivo na alta hospitalar }[n= \\
1.028]\end{array}$} \\
\hline Aleitamento materno exclusivo na alta & 955 & 92,9 & 59,8 & 1,79 & $1,32-2,42$ & 0,000 \\
\hline Não aleitamento materno exclusivo na alta & 73 & 7,1 & 33,5 & 1,00 & & \\
\hline \multicolumn{7}{|l|}{ Faixa etária do bebê (meses) [n = 1.029] } \\
\hline 아-1 & 121 & 11,8 & 82,1 & 2,39 & $1,84-3,12$ & 0,000 \\
\hline $1+2$ & 234 & 22,7 & 72,3 & 2,11 & $1,62-2,75$ & 0,000 \\
\hline $2+3$ & 205 & 19,9 & 59,7 & 1,74 & $1,32-2,29$ & 0,000 \\
\hline $3 \vdash-4$ & 193 & 18,8 & 53,3 & 1,56 & $1,18-2,07$ & 0,001 \\
\hline 4 ト5 & 159 & 15,5 & 38,5 & 1,12 & $0,82-154$ & 0,477 \\
\hline 5 -6 & 117 & 11,4 & 34,3 & 1,00 & & \\
\hline
\end{tabular}

IC95\%: intervalo de 95\% de confiança.

seu parto em Hospitais Amigos da Criança ou em processo de certificação e $63,7 \%$ delas tiveram parto normal. A grande maioria dos recém-nascidos $(92,9 \%)$ teve alta do hospital em aleitamento materno exclusivo. Das 1.029 crianças, $73,2 \%$ concentraram-se na faixa etária de 0 a 4 meses. $\mathrm{Na}$ análise bivariada, as variáveis sexo do bebê, tipo de parto, aleitamento materno exclusivo na alta hospitalar e idade do bebê alcançaram significância estatística observada menor ou igual a $20 \%$.

Quanto às características da unidade e orientações de aleitamento materno, $48 \%$ das mães estavam realizando o acompanhamento de seu bebê em unidades de pequeno porte, como postos de saúde, unidades de atenção de cuidados primários à saúde e PACS, $34 \%$ em centros munici- pais de saúde e $18 \%$ em unidades de grande porte como postos de assistência médica e policlínicas. A prevalência de aleitamento materno exclusivo foi semelhante nas três categorias de unidades de saúde avaliadas neste estudo, variando de $57,1 \%$ a 58,8\%, sem significância estatística. Receberam orientação sobre amamentação no primeiro dia em que o bebê veio à unidade básica $57,6 \%$ das mães. Foi falado sobre amamentação na consulta para $76,2 \%$ das mães, e em grupo, para $31,9 \%$. Relataram $49,8 \%$ das mães que foi mostrado como colocar o bebê para mamar, $65,3 \%$ que foi explicado sobre livre demanda e 72,4\% que foi falado até quando o aleitamento materno deve ser exclusivo. Receberam de 1 a 5 orientações sobre aleitamento materno $85,8 \%$ das mães. O percentual de $46,4 \%$ declarou que estava satisfeito com 
o apoio recebido na unidade para amamentar, $79,7 \%$ reportaram que o acompanhamento do bebê pela UBS era considerado ótimo ou bom. Na análise bivariada, alcançaram nível de significância observado menor ou igual a $20 \%$ as variáveis: orientação sobre amamentação no primeiro dia em que o bebê veio à unidade básica, ter sido falado sobre amamentação na consulta, ter sido informado sobre amamentação no grupo, ter sido mostrado como colocar o bebê para mamar, ter sido explicado sobre livre demanda, número de orientações recebidas na UBS e qualidade do acompanhamento do bebê segundo as mães (Tabela 3).

Os resultados da análise multivariada, obtidos pelo modelo final da regressão de Poisson, são apresentados na Tabela 4 . Nesse modelo final, as seguintes variáveis maternas apresentaram-se associadas com significância estatística observada menor ou igual a $5 \%$ ao aleitamento materno exclusivo: declarar-se de cor branca $(\mathrm{RP}=1,20$; IC95\%: 1,05-1,36); escolaridade alta $(\mathrm{RP}=1,19$; IC95\%: 1,05-1,35); ter companheiro $(\mathrm{RP}=1,72$; IC95\%: 1,02-2,90); experiência prévia com amamentação (RP = 1,27; IC95\%: 1,08-1,49) e alta hospitalar em aleitamento materno exclusivo (RP = 2,01; IC95\%: 1,20-3,36). As ações de promoção, proteção e apoio à amamentação que se mostraram associadas ao aleitamento materno exclusivo foram: ter sido falado sobre amamentação em grupo $(\mathrm{RP}=1,14$; IC95\%: 1,01-1,28) e ter sido mostrado como colocar o bebê para mamar ( $R P=1,20$; IC95\%: 1,08-1,33). A cada mês de vida do bebê, a prevalência de aleitamento materno exclusivo diminuiu $17 \%$ $(\mathrm{RP}=0,83$; IC95\%: 0,79-0,87).

\section{Discussão}

No presente estudo, a prevalência de aleitamento materno exclusivo entre crianças menores de seis meses assistidas pelas UBS do Município do Rio de Janeiro foi de 58,1\%. Apesar de esses resultados ainda estarem aquém das recomendações internacionais 4 , eles se mostraram superiores aos encontrados na pesquisa realizada na campanha de vacinação de 2006 no Município do Rio de Janeiro $(33,3 \%)$ com crianças da rede pública e particular 15 , e sinalizam a qualidade da produção do cuidado à criança assistida na rede básica de saúde, particularmente quanto às ações de promoção, proteção e apoio ao aleitamento materno. A rede básica de saúde do Município do Rio de Janeiro vem passando por um processo de qualificação na assistência ao aleitamento materno, com capacitação de equipes de saúde na IUBAAM 8. O Passo 10 da IUBAAM preconiza a implementação de grupos de apoio à amamentação com gestantes e mães e o Passo 6 as orientações no manejo da amamentação ${ }^{8}$. Segundo Rito 17 , a rede básica de saúde apresenta nível intermediário de implantação desta iniciativa e várias unidades adotam práticas de apoio à amamentação.

Ficou demonstrado neste estudo que alguns grupos populacionais apresentam maior prevalência de aleitamento materno exclusivo. Declarar-se de cor branca aumentou em $20 \%$ a prevalência de aleitamento materno exclusivo. Segundo dados da PNDS de 1996 e 2006 13, as medianas em meses do aleitamento materno exclusivo no Brasil são mais elevadas entre as mulheres de cor branca, apontando a necessidade de pesquisas que identifiquem intervenções que minorem a introdução precoce de outros alimentos na alimentação das crianças não brancas nos primeiros seis meses de vida.

A associação entre a escolaridade materna alta e a duração do aleitamento materno exclusivo mostrou significância estatística neste estudo ( $p=0,008$ ), apontando que mães de escolaridade mais baixa tendem a introduzir mais precocemente alimentos, em concordância com outros trabalhos 25,26. Bueno et al. 26, em um estudo de coorte em São Paulo, demonstrou que a escolaridade materna até o ensino fundamental representa o dobro do risco em relação ao nível superior para introdução de outros alimentos antes dos seis meses. Este achado poderia estar relacionado ao conhecimento sobre os benefícios do aleitamento materno exclusivo contribuindo para que a mulher de escolaridade mais alta venha a fazer a opção pelo aleitamento materno exclusivo nos primeiros seis meses de vida do bebê, como é recomendado 4 .

Ter companheiro aumentou a prevalência de aleitamento materno exclusivo em $72 \%$. Um estudo realizado em países da América Latina sinalizou que, no Brasil, a duração do aleitamento materno exclusivo tendia a ser maior quando o pai do bebê morava com a família $(p=0,08) 27$. Em ensaio controlado, na Itália, as orientações fornecidas aos pais e companheiros sobre como resolver problemas com a amamentação elevaram a prevalência de aleitamento materno exclusivo 28 , sugerindo que este tipo de intervenção deveria ser desenvolvida nos serviços. Segundo nosso estudo, maior atenção deve ser dada às mulheres que não têm companheiro.

Ter tido experiência anterior com amamentação por mais de seis meses aumentou em $27 \%$ a prevalência de amamentação exclusiva nos primeiros seis meses de vida. A experiência prévia com a amamentação tem sido associada com uma duração maior da amamentação e do aleita- 
Tabela 3

Prevalência e razão de prevalência (RP) bruta de aleitamento materno exclusivo segundo características da unidade básica de saúde (UBS) e das orientações de amamentação oferecidas às mães das crianças menores de seis meses assistidas nas UBS do Município do Rio de Janeiro, Brasil, 2007.

\begin{tabular}{|c|c|c|c|c|c|c|}
\hline \multirow[t]{2}{*}{ Variáveis } & \multicolumn{2}{|c|}{ Freqüência } & \multicolumn{4}{|c|}{ Aleitamento materno exclusivo } \\
\hline & $\mathbf{n}$ & $\%$ & $\%$ & RP bruta & IC95\% & $\begin{array}{l}\text { Valor } \\
\text { de p }\end{array}$ \\
\hline \multicolumn{7}{|l|}{ Tipo de unidade $[\mathrm{n}=1.029]$} \\
\hline Centros municipais de saúde & 350 & 34,0 & 58,3 & 1,02 & $0,91-115$ & 0,728 \\
\hline Postos de assistência médica/Policlínica & 185 & 18,0 & 58,8 & 1,03 & $0,89-1,19$ & 0,667 \\
\hline Postos de saúde/Unidades de atenção de cuidados primários à saúde/ & 494 & 48,0 & 57,1 & 1,00 & & \\
\hline \multicolumn{7}{|l|}{ Programa de agentes comunitários de saúde } \\
\hline \multicolumn{7}{|l|}{ Orientação sobre amamentação no 1o dia em que veio à UBS [n = 975] } \\
\hline Sim & 562 & 57,6 & 54,1 & 0,89 & $0,77-1,03$ & 0,126 \\
\hline Não ou apenas foi marcada palestra & 413 & 42,4 & 60,7 & 1,00 & & \\
\hline \multicolumn{7}{|l|}{ Aqui nesta unidade falaram sobre amamentação na consulta [n = 668] } \\
\hline Sim & 509 & 76,2 & 53,7 & 0,85 & $0,69-1,04$ & 0,133 \\
\hline Não & 159 & 23,8 & 63,2 & 1,00 & & \\
\hline \multicolumn{7}{|l|}{ Aqui nesta unidade falaram sobre amamentação em grupo [ $n=668]$} \\
\hline Sim & 213 & 31,9 & 62,3 & 1,17 & $1,04-1,32$ & 0,016 \\
\hline Não & 455 & 68,1 & 53,1 & 1,00 & & \\
\hline \multicolumn{7}{|l|}{ Aqui nesta unidade falaram sobre amamentação de outro jeito [ $\mathrm{n}=668$ ] } \\
\hline Sim & 53 & 7,9 & 50,3 & 0,89 & $0,61-1,31$ & 0,532 \\
\hline Não & 615 & 92,1 & 56,4 & 1,00 & & \\
\hline \multicolumn{7}{|l|}{ Mostraram como colocar o bebê para mamar $[n=1.025]$} \\
\hline Sim & 510 & 49,8 & 60,0 & 1,08 & $0,97-1,19$ & 0,160 \\
\hline Não & 515 & 50,2 & 55,8 & 1,00 & & \\
\hline \multicolumn{7}{|l|}{ Explicaram que o bebê deve mamar quando quiser $[n=1.028]$} \\
\hline $\operatorname{Sim}$ & 671 & 65,3 & 55,2 & 0,88 & $0,78-1,00$ & 0,058 \\
\hline Não & 357 & 34,7 & 62,6 & 1,00 & & \\
\hline \multicolumn{7}{|l|}{ Explicaram como tirar leite do peito $[n=1.027]$} \\
\hline Sim & 440 & 42,8 & 58,1 & 1,01 & $0,90-1,13$ & 0,877 \\
\hline Não & 587 & 57,2 & 57,6 & 1,00 & & \\
\hline \multicolumn{7}{|l|}{ Falaram que não se deve dar mamadeira [n=1.028] } \\
\hline $\operatorname{Sim}$ & 713 & 69,4 & 57,5 & 0,98 & $0,83-1,16$ & 0,814 \\
\hline Não & 315 & 30,6 & 58,6 & 1,00 & & \\
\hline \multicolumn{7}{|l|}{ Falaram até quando deve mamar só no peito [ $n=1.009]$} \\
\hline Sim, por 6 meses & 731 & 72,4 & 56,7 & 0,95 & $0,87-1,03$ & 0,213 \\
\hline Não ou por período diferente de 6 meses & 278 & 27,6 & 59,9 & 1,00 & & \\
\hline \multicolumn{7}{|l|}{ Recebeu alguma orientação sobre amamentação [n = 1.029] } \\
\hline $1-5$ & 883 & 85,8 & 56,7 & 0,87 & $0,80-0,95$ & 0,007 \\
\hline Nenhuma & 146 & 14,2 & 65,1 & 1,00 & & \\
\hline \multirow{2}{*}{\multicolumn{7}{|c|}{$\begin{array}{l}\text { Satisfação das mães com o apoio para amamentar nas unidades básicas } \\
{[n=1.028]}\end{array}$}} \\
\hline & \multicolumn{6}{|c|}{$[n=1.028]$} \\
\hline Sim/Mais ou menos & 658 & 64,0 & 57,5 & 0,98 & $0,83-1,15$ & 0,781 \\
\hline Não & 370 & 36,0 & 58,4 & 1,00 & & \\
\hline \multicolumn{7}{|l|}{ Acompanhamento do bebê $[n=1.028]$} \\
\hline Ótimo/Bom & 820 & 79,7 & 59,6 & 1,18 & $1,00-1,40$ & 0,042 \\
\hline Mais ou menos/Ruim/Péssimo & 208 & 20,3 & 50,4 & 1,00 & & \\
\hline
\end{tabular}

IC95\%: intervalo de 95\% de confiança. 
Razão de Prevalência bruta e ajustada, segundo características das mães, variáveis da assistência e ações de aleitamento materno desenvolvidas com as mães das crianças menores de seis meses assistidas nas unidades básicas de saúde (UBS) do Município do Rio de Janeiro, Brasil, 2007 ( $n=664$ ).

\begin{tabular}{|c|c|c|c|c|}
\hline Variáveis & RP bruta & RP ajustada & IC95\% & Valor de $\mathrm{p}$ \\
\hline \multicolumn{5}{|l|}{ Cor } \\
\hline Branca & 1,11 & 1,20 & $1,05-1,36$ & 0,008 \\
\hline Não branca & 1,00 & 1,00 & & \\
\hline \multicolumn{5}{|l|}{ Escolaridade } \\
\hline Ensino Médio completo ou mais & 1,11 & 1,19 & $1,05-1,35$ & 0,008 \\
\hline Ensino Médio incompleto & 1,00 & 1,00 & & \\
\hline \multicolumn{5}{|l|}{ Situação conjugal } \\
\hline Tem companheiro & 1,47 & 1,72 & $1,02-2,90$ & 0,040 \\
\hline Não tem companheiro & 1,00 & 1,00 & & \\
\hline \multicolumn{5}{|l|}{ Amamentação pregressa (meses) } \\
\hline $7-84$ & 1,19 & 1,27 & $1,08-1,49$ & 0,005 \\
\hline $0-6$ e 29 dias & 1,00 & 1,00 & & \\
\hline \multicolumn{5}{|c|}{ Aleitamento materno exclusivo na alta hospitalar } \\
\hline Sim & 1,79 & 2,01 & $1,20-3,36$ & 0,010 \\
\hline Não & 1,00 & 1,00 & & \\
\hline \multicolumn{5}{|l|}{ Falaram sobre amamentação em grupo } \\
\hline Sim & 1,17 & 1,14 & $1,01-1,28$ & 0,040 \\
\hline Não & 1,00 & 1,00 & & \\
\hline \multicolumn{5}{|c|}{ Mostraram como colocar o bebê para mamar } \\
\hline $\operatorname{Sim}$ & 1,08 & 1,20 & $1,08-1,33$ & 0,001 \\
\hline Não & 1,00 & 1,00 & & \\
\hline Idade do bebê & - & 0,83 & $0,79-0,87$ & 0,000 \\
\hline
\end{tabular}

IC95\%: intervalo de 95\% de confiança.

mento materno exclusivo 29. Este achado reforça a importância de um apoio maior às mulheres que ainda não amamentaram ou que amamentaram por pouco tempo seus filhos anteriormente.

A alta hospitalar em aleitamento materno exclusivo foi a variável que mais aumentou a prevalência de aleitamento materno exclusivo nos primeiros seis meses de vida. Ter recebido alta do hospital em aleitamento materno exclusivo dobrou a prevalência de aleitamento materno exclusivo nos primeiros seis meses de vida do bebê, sinalizando a efetividade das ações de promoção, proteção e apoio à amamentação desenvolvidas nos hospitais e o sinergismo entre as ações realizadas nos hospitais e unidades básicas de saúde, já demonstrado em outros trabalhos 25. Em nosso estudo não foi encontrada associação entre o desfecho e o fato de a criança ter nascido em Hospital Amigo da Criança ou em hospital em processo de se tornar Hospital Amigo da Criança. No entanto, a prevalência de $92,9 \%$ de aleitamento materno exclusivo encontrada na alta hospitalar revela o quanto as práticas de apoio à amamentação estão disseminadas nos vários hospitais do Município do Rio de Janeiro.

Neste estudo, ter sido mostrado como colocar o bebê para mamar aumentou a prevalência de aleitamento materno exclusivo em $20 \%$. Este dado é consistente com outros estudos 30 que demonstram que a técnica correta de amamentar contribui para o estabelecimento de um padrão de sucção efetiva do leite materno pelo bebê, para o ganho ponderal adequado e para a prevenção de traumas mamilares e mastites e que esta orientação deve fazer parte da assistência às mães e bebês. Estudo de Caldeira et al. 19 demonstrou que uma intervenção de capacitação de profissionais de saúde na IUBAAM, com abordagem de questões práticas relacionadas ao manejo clínico da amamentação, repercutiu positivamente na curva de sobrevida do aleitamento materno exclusivo.

No presente estudo, 31,9\% das mães relataram ter sido falado sobre amamentação em grupo nas unidades básicas e esta variável aumentou a prevalência de aleitamento materno 
exclusivo em $14 \%$, enquanto ter recebido orientação individual, na consulta, não mostrou associação significativa com o aleitamento materno exclusivo. Alguns trabalhos na literatura têm evidenciado a importância de os grupos de apoio à amamentação aumentarem a prevalência do aleitamento materno exclusivo e a satisfação das mulheres com o apoio recebido. Guise et al. 18 buscaram avaliar por intermédio de uma revisão sistemática quais intervenções desenvolvidas nos serviços de saúde, aumentavam o início e a duração da amamentação. O estudo apontou os trabalhos educativos desenvolvidos em grupo com gestantes no pré-natal como a intervenção mais efetiva em aumentar o início e a duração da amamentação até os três meses. Os grupos se caracterizavam por oferecer informações sobre os benefícios do leite materno, orientações práticas no manejo da amamentação como posicionamento e pega correta do bebê na mamada, além de esclarecer dúvidas em amamentação. Questiona, contudo, o quanto tais ações educativas estão acessíveis nos diversos serviços de saúde e que barreiras existem para sua implementação.

Hoddinott et al. 31 avaliaram em um estudo de intervenção quantitativo e qualitativo as ações desenvolvidas pelos profissionais de saúde na atenção básica e sua participação em atividades de grupo envolvendo gestantes e mães. Eles comprovaram a efetividade dos grupos em aumentar a prevalência de aleitamento materno e a satisfação das mulheres com a intervenção. A intervenção de grupo foi mais efetiva do que a orientação individual, especialmente nas áreas onde os profissionais trabalhavam juntos, integrados, no mesmo espaço, e o vínculo entre os profissionais era forte. A intervenção aumentou a prevalência de amamentação até duas semanas de vida em $6,8 \%$, comparada com o declínio da amamentação no resto da Escócia de 0,4\%. Um outro estudo qualitativo ${ }^{32}$, realizado por estes pesquisadores em 2006, revelou que as atividades de grupo eram escolhidas pelas mulheres na Escócia, porque propiciavam uma diversidade de experiências, possibilitavam às mulheres assistidas tomar decisões em relação à amamentação por si mesmas e ofereciam um lugar seguro para juntas praticarem a amamentação. A orientação individual foi percebida como de maior risco para a auto-estima e para o empoderamento do que as orientações oferecidas em grupo.

Segundo Franco et al. 33, esta questão é particularmente importante para a compreensão da organização da assistência à saúde e traz em seu bojo um potencial transformador por levar à reflexão sobre o processo de trabalho e as práticas de cuidado. Verifica-se, segundo os autores, que no modelo assistencial vigente em nosso país, a distribuição do trabalho nos serviços de saúde é dimensionada para concentrar o fluxo da assistência no trabalho médico e que as atividades de grupo e interdisciplinares que envolvem outros profissionais são pouco valorizadas. Observam que há um potencial de trabalho de todos os profissionais que poderia ser aproveitado para cuidados diretos com o usuário, elevando assim a capacidade resolutiva dos serviços de forma a qualificar o processo de trabalho e a produção do cuidado.

Com preocupações dessa natureza, muitas iniciativas vêm sendo desenvolvidas no Brasil a fim de reconstruir as práticas de saúde, dando relevância a esta ótica do cuidado em saúde, valorizando a escuta, o vínculo e a responsabilização na organização da assistência na atenção básica 33. Os estudos de Guise et al. 18 e Hoddinott et al. 31 apontam que os grupos de apoio à amamentação, para gestantes e mães, constituem um espaço de atuação interdisciplinar, com troca de experiências e vivências entre trabalhadores de saúde e mães, além de maior escuta de suas necessidades, levando à melhoria da produção do cuidado e maior resolubilidade à rede básica de saúde.

Vale ressaltar que há algumas limitações no presente estudo. Trata-se de estudo transversal, sujeito a viés de memória, pois foram obtidos dados sobre as ações desenvolvidas nas unidades básicas mediante perguntas realizadas nas entrevistas às mães. Mães que apresentaram maior dificuldade no processo da amamentação podem ter se recordado mais das orientações recebidas, contribuindo assim para minorar a força da associação dessas ações com o aleitamento materno exclusivo. Além disso, mães com dificuldades podem ter recebido maior número de orientações e, mesmo assim, não estar em aleitamento materno exclusivo.

Este trabalho sinaliza que atenção especial deve ser dada aos grupos populacionais que apresentaram menor prevalência de aleitamento materno exclusivo na atenção básica do $\mathrm{Mu}$ nicípio do Rio de Janeiro, como as mulheres de cor não branca, escolaridade baixa e aquelas que não têm companheiro. As orientações sobre o manejo da amamentação e os grupos de apoio à amamentação, conduzidos nas UBS, mostraramse associados positivamente à prevalência do aleitamento materno exclusivo. Recomenda-se que essas ações sejam implementadas no conjunto das UBS e que iniciativas que valorizem o aleitamento materno na atenção básica, como a Unidade Básica Amiga da Amamentação, sejam fortalecidas. 


\section{Resumo}

Este estudo teve o objetivo de analisar a associação en tre ações de promoção, proteção e apoio à amamentação realizadas em unidades básicas de saúde (UBS) e a prevalência de aleitamento materno exclusivo nos primeiros seis meses de vida. Estudo transversal realizado em amostra representativa de 1.029 mães de crianças menores de seis meses assistidas em unidades básicas de saúde do Município do Rio de Janeiro, Brasil. As razões de prevalência (RP) foram obtidas por regressão de Poisson. A prevalência de aleitamento materno exclusivo foi de 58,1\%. Estiveram associadas à maior prevalência de aleitamento materno exclusivo: a cor branca $(R P=1,20$; IC95\%: 1,05-1,36) e a escolaridade alta $(R P=1,19$; IC95\%: 1,05-1,35); ter companheiro $(R P=1,72 ;$ IC95\%: 1,02-2,90); experiência prévia com amamentação ( $R P=1,27$; IC95\%: 1,08-1,49); alta hospitalar em aleitamento materno exclusivo $(R P=2,01$; IC95\%: 1,20-3,36; ter recebido orientação em grupo $(R P=1,14$; IC95\%: 1,01-1,28); e ter sido mostrado como amamentar (RP = 1,20; IC95\%: 1,08-1,33). A prevalência de aleitamento materno exclusivo diminuiu $17 \%$ a cada mês de vida do bebê. Os grupos de apoio à amamentação e a orientação sobre seu manejo contribuíram para o aleitamento materno exclusivo na atenção básica.

Aleitamento Materno; Atenção Primária à Saúde; Es tudos Transversais

\section{Colaboradores}

R. S. V. Pereira foi responsável principal pela análise e interpretação dos dados, redação do artigo e aprovação final da versão a ser publicada. M. I. C. Oliveira colaborou na análise e interpretação dos dados, na redação do artigo e na aprovação da versão final do artigo a ser publicado. C. L. T. Andrade e A. S . Brito colaboraram na análise e interpretação dos dados e na revisão crítica do conteúdo do artigo, e da aprovação final da versão a ser publicada.

\section{Referências}

1. Giugliani ERJ. Amamentação: como e por que promover. J Pediatr (Rio J.) 1994; 70:138-51.

2. Gartner LM, Morton J, Lawrence RA, Naylor AJ, O'Hare D, Schanler RJ, et al. Breastfeeding and the use of human milk. Pediatrics 2005; 115:496-506.

3. WHO Multicentre Growth Reference Study Group. WHO Child Growth Standards based on length/ height, weight and age. Acta Paediatr Suppl 2006; 450:76-85.

4. World Health Organization. Report of an expert consultation on the optimal duration of exclusive breastfeeding. Geneva: World Health Organization; 2001.

5. World Health Organization. Indicators for assessing infant and young child feeding practices: conclusions of a consensus. Washington DC: World Health Organization; 2008.

6. Saadeh R, Akré J. Ten steps to successful breastfeeding: a summary of the rationale and scientific evidence. Birth 1996; 23:154-60.
7. World Health Organization/United Nations Children's Fund. The global criteria for the Baby Friendly Hospital Initiative. Geneva: World Health Organization/New York: United Nations Children's Fund; 1992.

8. Oliveira MIC, Camacho LAB, Tedstone AE. Extending breastfeeding duration through primary care: a systematic review of prenatal and postnatal interventions. J Hum Lact 2001; 17:326-43.

9. Secretaria de Estado de Saúde do Rio de Janeiro. Resolução SES no. 2.673 de 2 de março de 2005. Implanta a Iniciativa Unidade Básica Amiga da Amamentação no Estado do Rio de Janeiro e dá outras providências. Diário Oficial da União 2005; 14 mar.

10. Hannula L, Kaunonen M, Tarkka MT. A systematic review of professional support interventions for breastfeeding. J Clin Nurs 2008; 17:1132-43. 
11. Ministério da Saúde. Política Nacional de Promo ção, Proteção e Apoio ao Aleitamento Materno. http://portal.saude.gov.br/portal/saude/cidadao/ visualizar_texto.cfm?idtxt=26350. (acessado em 08/Fev/2010).

12. Black RE, Morris SS, Bryce J. Where and why are 10 million children dying every year? Lancet 2003; 361:2226-34

13. Ministério da Saúde. Pesquisa Nacional de Demografia e Saúde da Criança e da Mulher - PNDS2006: dimensões do processo reprodutivo e da saúde da criança. Brasília: Ministério da Saúde/ Centro Brasileiro de Saúde e Planejamento; 2008.

14. Ministério da Saúde. II Pesquisa de prevalência de aleitamento materno nas capitais brasileiras e Distrito Federal, 2008. http://bvsms.saude.gov. $\mathrm{br} / \mathrm{bvs} /$ publicacoes/pesquisa_prevalencia_aleita mento_materno.pdf (acessado em 10/Dez/2009).

15. Castro IRR, Engstrom EM, Cardoso LO, Damião JJ, Rito RVF, Gomes MASM. Tendência temporal da amamentação na cidade do Rio de Janeiro: 19962006. Rev Saúde Pública 2009; 43:1021-9.

16. Agência Nacional de Vigilância Sanitária. Banco de leite humano: funcionamento, prevenção e controle de riscos. Brasília: Agência Nacional de Vigilância Sanitária, Ministério da Saúde; 2008.

17. Rito RVVF. Iniciativa Unidade Básica Amiga da Amamentação: avaliação em unidades da rede básica de saúde da cidade do Rio de Janeiro [Tese de Doutorado]. Rio de Janeiro: Instituto Fernandes Figueira, Fundação Oswaldo Cruz; 2009.

18. Guise JM, Palda V, Westhoff C, Chan BK, Helfand $\mathrm{M}$, Lieu TA, et al. The effectiveness of primary care base interventions to promote breastfeeding: systematic evidence review and meta-analysis for the US Preventive Services Task Force. Ann Fam Med 2003; $1: 70-8$

19. Caldeira AP, Fagundes GC, Aguiar GN. Intervenção educacional em equipes do Programa de Saúde da Família para promoção da amamentação. Rev Saúde Pública 2008; 42:1027-33.

20. Levi PS, Lemeshow S. Sampling of populations: methods and applications, textbook and solutions manual. New York: Wiley-Interscience; 2003.

21. Aarts C, Kylberg E, Hornell A, Hofvander Y, GebreMedhin M, Greiner T. How exclusive is exclusive breastfeeding? A comparison of data since birth with current status data. Int J Epidemiol 2000; 29:1041-6.

22. Ministério da Saúde. Recomendações para profilaxia da transmissão materno-infantil do HIV e terapia anti-retroviral. Brasília: Ministério da Saúde; 2001.
23. Coutinho LMS, Scazufca M, Menezes PR. Métodos para estimar razão de prevalência em estudos de corte transversal. Rev Saúde Pública 2008; 42:992-8.

24. Szwarcwald CL, Damacena GN. Amostras complexas em inquéritos populacionais: planejamento e implicações na análise estatística dos dados. Rev Bras Epidemiol 2008; 11 Suppl 1:38-45.

25. Venâncio SI, Escuder MML, Kitoko P, Rea MF, Monteiro CA. Freqüência e determinantes do aleitamento materno em municípios do Estado de São Paulo. Rev Saúde Pública 2002; 36:313-8.

26. Bueno MB, Souza JMP, Souza SB, Paz SMRS, Siqueira AAF. Riscos associados ao processo de desmame entre crianças nascidas em hospital universitário de São Paulo entre 1998 e 1999: estudo de coorte prospectivo do primeiro ano de vida. Cad Saúde Publica 2003; 19:1453-60.

27. Pérez-Scamilla R, Lutter C, Segall AM, Rivera A, Trevino-Siller S, Sanghvi T. Exclusive breast-feeding duration is associated with attitudinal, socioeconomic and biocultural determinants in three latin american countries. J Nutr 1995; 125:2972-84.

28. Pisacane A, Continisio GI, Aldinucci M, Amora S, Continisio P. A controlled trial of the father's role in breastfeeding promotion. Pediatrics 2005; 116:e494-8.

29. Bakoula C, Veltsista A, Prezerakou A, Moustaki M, Fretzayas A, Nicolaidou P. Working mothers breastfeed babies more than housewives. Acta Paediatr 2007; 96:510-5.

30. Weigert EML, Giugliani ERJ, França MCT, Oliveira LD, Bonilha A, Espírito Santo LC, et al. Influência da técnica de amamentação nas freqüências de aleitamento materno exclusivo e lesões mamilares no primeiro mês de lactação. J Pediatr (Rio J.) 2005; 81:310-6.

31. Hoddinott P, Pill R, Chalmers M. Health professionals, implementation and outcomes: reflections on a complex intervention to improve breastfeeding rates in primary care. Fam Prat 2007; 24:84-91.

32. Hoddinott P, Phil M, Chalmers M, Pill R. One-toone or group-based peer support for breastfeeding? Women's perceptions of a breastfeeding peer coaching intervention. Birth 2006; 33:139-46.

33. Franco TB. Produção do cuidado e produção pedagógica: cenários de integração do sistema de saúde no Brasil. Interface Comun Saúde Educ 2007; 11:427-38

Recebido em 14/Abr/2010

Versão final reapresentada em 02/Ago/2010

Aprovado em 18/Ago/2010 